\title{
Dokumentation
}

\section{Heinrich Goebel - Aufstieg und Fall einer deutschen Legende \\ Zum Erscheinen eines neuen Buches über den Erfinder der Glühlampe}

\author{
von Frank DitTMann
}

\section{Eine Provinzstadt in Aufruhr}

In Springe, einem Städtchen in der Nähe von Hannover, kochen die Emotionen hoch. Seit Jahren wirbt die Stadt mit ihrem großen Sohn Heinrich Goebel, der lange vor Edison die Glühlampe erfunden haben soll. Weithin sichtbar leuchtet jeden Abend ein Glühlampensymbol auf der Göbelbastei am Deisterhang. Nun aber wird alles in Frage gestellt, ${ }^{1}$ und zwar durch eine historische Dissertation, die seit Januar 2007 gedruckt vorliegt. ${ }^{2}$ Autor ist Hans-Christian Rohde, der Germanistik, Geschichte und Politikwissenschaft studierte und heute am Gymnasium in Springe lehrt. Den Anstoß zu dieser Arbeit gab das falsche Sterbedatum von Heinrich Goebel, das allerorten zu finden ist, so auch auf zwei Gedenktafeln in Springe. Rohde suchte in den Archiven intensiv nach historisch gesicherten Fakten. Dabei konnte er auch die Dokumente jener Patentprozesse in den USA auswerten, in die Goebel in den 1890er Jahren verwickelt war. Was Rohde bei seiner Suche zu Tage förderte, resümiert er folgendermaßen: „Die 1923 ausgestaltete Geschichte von Heinrich Göbel als dem Erfinder der Glühlampe wird mit der Stadt Springe verbunden bleiben. [...] Wer sie jedoch künftig erzählen möchte, sollte redlich sein und sie als Legende kennzeichnen." ${ }^{\text {3 }}$

Nach diesem ernüchternden Ergebnis sind ansässige und ehemalige Bürger der Stadt gespalten. Einige verlangen, dass nicht länger der Name eines „Hochstaplers“ im Schulnamen über ihrem Abgangszeugnis stehe. Andere wiederum wollen das Idol nicht einfach kampflos stürzen lassen. So werden Briefe an das Deutsche Museum in München geschrieben, damit dieses als „Bezeugungsinstanz“ die Sachlage letztlich kläre. Auch Journalisten melde-

1 Die Diskussion dauert schon etwas länger an. 2000 begann das Museum auf dem Burghof in Springe mit der Quellensuche und seit dieser Zeit setzt sich auch ein „Göbel-Stammtisch" mit dem Thema auseinander. Im Frühjahr 2006 machte der Künstler Dietmar Moews mit einem Vortrag in Springe die Zweifel an Goebels Erfindungspriorität einer größeren Öffentlichkeit zugänglich. Siehe die Meldung „Heinrich Göbel wird zurückgestuft“, in: Neue Deister Zeitung vom 11.03.2006, S. 11. Moews verbreitet seine Sicht auch in der von ihm herausgegebenen Zeitschrift Neue Sinnlichkeit 26, Dez. 2005.

2 Hans-Christian Rohde, Die Göbel-Legende. Der Kampf um die Erfindung der Glühlampe, Springe 2007.

3 Ebd., S. 135. 
ten sich beim Autor dieses Beitrags. Erstaunt, ob der plötzlichen Aktualität der Technikgeschichte, deren Existenz oft genug nicht zur Kenntnis genommen wird, versuchte er sich selbst ein Bild von der Sachlage zu verschaffen. Interessant ist dabei, wie drei Geschichten unentwirrbar ineinander verwoben sind: Da ist die Lebensgeschichte von Heinrich/Henry Goebel, bzw. das, was wir heute aus den Akten entnehmen können. Davon verselbständigt hat sich die Legende vom deutschen Erfinder der Glühlampe. Auf einer dritten Ebene geht es um den Umgang einer Stadt mit dem ernüchternden Faktum, dass eine über 80 Jahre angenommene Tatsache mit hoher Sicherheit falsch ist. Die Aktualität, den ein technikgeschichtlicher Sachverhalt angenommen hat, und die Tatsache, dass die Goebel-Legende in der Vergangenheit auch in historischen und elektrotechnischen Fachzeitschriften ein Sprachrohr fand, scheint Anlass genug, das kürzlich erschienene Buch ausführlich vorzustellen.

\section{Leben und Werk Heinrich Goebels}

Heinrich (Henry) Goebel, geboren am 20. April 1818 in Springe, gestorben am 16. Dezember 1893 in New York, ,erfand 1854 (vor T.A. Edison) eine elektrische Glühlampe mit einem Glühfaden aus verkohlter Bambusfaser. [...] Nach 12-jährigem Rechtsstreit wurde G. die Priorität für die Erfindung der Glühlampe zugesprochen. "“4 So kann man es in der neuesten Brockhaus Enzyklopädie nachlesen. Und so oder so ähnlich steht es seit fast 80 Jahren auch in vielen anderen deutschen Nachschlagewerken, während Goebel außerhalb des deutschen Sprachraumes weitgehend unbekannt ist. Dennoch ist von diesen Angaben lediglich das Geburtsdatum richtig. Was Rohde bei seinen ausgiebigen Quellenstudien herausfand, sei an dieser Stelle referiert.

Unstrittig ist, dass Johann Heinrich Christoph Conrad Goebel am 20. April 1818 in Springe geboren und 1832 konfirmiert wurde. Ein Zeugnis bewertet die Fähigkeiten und Kenntnisse des 14-Jährigen im Lesen, Schreiben, Rechnen und in Religion als mangelhaft, entschuldigt dies aber mit langer Krankheit. Außerdem wird angemerkt: ,Scheint einen erfinderischen Geist zu haben. ${ }^{\text {"5 }}$ Diese Aussage wurde später als erster Beleg für Goebels überragenden ingeniösen Geist angesehen. Rohde hält diese Interpretation keineswegs für eindeutig, denn unmittelbar daneben verweist eine Bemerkung auf eine leichtsinnige sittliche Verfassung. Man könne auch annehmen, so Rohde, dass der Lehrer damit Goebels Eigenschaft herausstellen wollte, gerne Geschichten, Ausflüchte oder auch Lügen zu erfinden.

1834 begann Heinrich Goebel in Springe eine Schlosserlehre, machte sich 1837 selbstständig und heiratete 1844. Im November 1848 wanderte er mit seiner Familie in die USA aus und eröffnete in New York ein Uhrenge-

4 Goebel, Heinrich. In: Brockhaus Enzyklopädie in 30 Bänden, Leipzig, Mannheim ${ }^{21} 2006$, Bd. 11, S. 116. Ähnliche Angaben auch unter dem Stichwort „Glühlampe“ ebd., S. 92.

5 Rohde (wie Anm. 2), S. 61. 


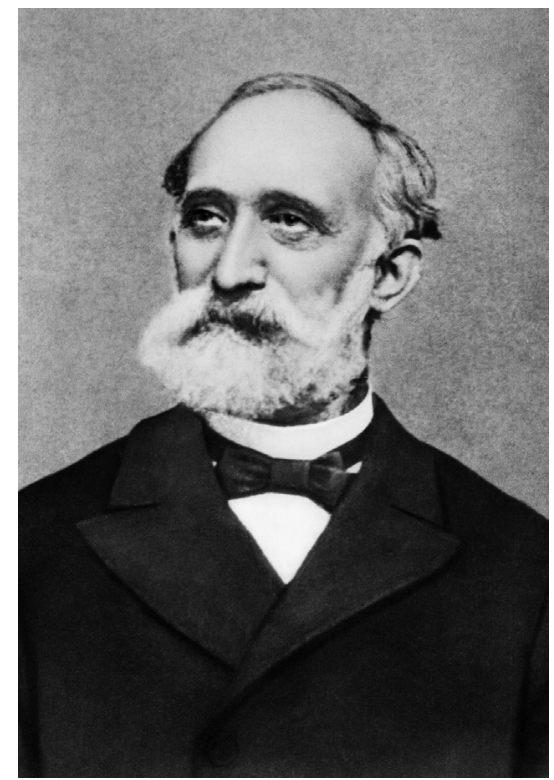

Abb. 1: Henry Goebel (1818-1893). Quelle: Deutsches Museum, München. Kürzlich wurde im Archiv des Deutschen Museums auch das Foto seiner Ehefrau Sophie Betty Julie Lübke, genannt Rodewig, identifiziert.

schäft. ${ }^{6}$ Einen Zusammenhang mit den revolutionären Ereignissen jener Jahre konnte Rohde nicht finden, er hält wirtschaftliche Gründe für die Übersiedlung für wahrscheinlich. 1852 beantragte Goebel die amerikanische Staatsbürgerschaft, die er 1865 erhielt. Der Autor des neuen Goebel-Buches fördert weitere Details der New Yorker Zeit zu Tage, die hier nicht ausgebreitet werden können. Dass Goebel durchaus technische Kreativität besaß und auch mit dem Rechtsgefüge der USA zumindest einigermaßen vertraut war, beweist sein Patent von 1865 zu einem Mechanismus, der das Säumen von Stoff mit Nähmaschinen erleichterte. Mehrfach bezeugt ist eine Geschichte, die später in der Goebel-Legende wieder auftauchen sollte. Zwischen 1856 und 1875 zog er nachts mit einem Wagen durch die Straßen und bot Passanten gegen einige Cent einen Blick auf den Sternenhimmel durch ein darauf montiertes Fernrohr an. Im Gegensatz zu späteren Aussagen fand Rohde jedoch keinen Hinweis auf die Verwendung von Glühlampen als Werbemittel. Erst nach $1880 \mathrm{kam}$ Goebel nachweislich damit in Berührung. Ab September 1881 arbeitete er für ein halbes Jahr bei der American Light Company. Dort hatte man Probleme mit der Befestigung der Kohlefäden an den $\mathrm{Zu}-$ leitungsdrähten und suchte einen Uhrmacher, der in der Lage war, die nötigen kleinen Klemmen herzustellen. Genau diesem Problem widmete sich

6 In den New Yorker Adressbüchern ist seit 1854/55 der Eintrag zu finden: „Goebel, Henry, watches, 271 1/2 Monroe Street“, vgl. Rohde (wie Anm. 2), S. 65; Goebel zog später mehrfach um. 
Goebel in einem Patent. ${ }^{7}$ Unser Protagonist löste bald die Verbindung zum Unternehmen und bemühte sich nun selbst um Geldgeber. Dazu sollten wohl auch Presseberichte dienen. So berichtete die New York Times vom 30. April 1882, dass Henry Goebel acht von ihm erfundene elektrische Lampen vorgeführt habe. Den Reportern hatte er mitgeteilt, dass er sich seit 30 Jahren mit Elektrizität beschäftige und bereits vor 29 Jahren ein elektrisches Licht erfunden habe. Am Schluss wird behauptet, er besitze eine Anzahl von Patenten, die alle wesentlichen Punkte zur Produktion von Glühlampen abdecken würden. ${ }^{8}$

Derlei offensichtliche Falschaussagen hätten normalerweise keine weiteren Auswirkungen gehabt, aber Mitte der 1880er Jahre begann Thomas Alva Edison, gegen den unautorisierten Nachbau seiner patentierten Glühlampen vorzugehen. In diesem Kontext wurde die Aussage, jemand habe lange vor dem Edison-Patent eine Glühlampe entwickelt, plötzlich interessant - und sei es auch nur, um mit allerlei juristischen Winkelzügen einen vom Patentinhaber angestrengten Prozess zu verzögern.

\section{Die Patentprozesse Anfang der 1890er Jahre}

Wie so oft bei der historischen Aufarbeitung von Innovationen, kann man auch für die Glühlampe keinen eindeutigen Erfinder ausmachen. Über zwei Dutzend Personen hatten zwischen 1820 und 1880 an der praktischen Nutzung des elektrischen Glühlichtes gearbeitet. Bekannt wurden davon nur wenige. ${ }^{9}$ Wichtig in unserem Zusammenhang ist, dass Edison am 4. November 1879 ein Patent für eine Glühlampe anmeldete, dessen Anspruch sich auf drei wesentliche Merkmale bezog. Ein Kohlefaden (1) brennt im Vakuum (2) und wird von Außen durch den Glaskörper mit Zuleitungsdrähten aus Platin (3) versorgt. ${ }^{10}$ Neben vielen Detailverbesserungen an der Glühlampe selbst entwickelte Edison mit seinem Team auch die nötigen Elemente eines kompletten Beleuchtungssystems: Fassungen, Schalter, Sicherungen, Leitungen, Dynamomaschinen, Stromzähler und anderes Zubehör. Bis zum Herbst 1886 besaß die Edison Electric Light Company 266 Patente für ihr Beleuchtungssystem und es war klar, dass man kaum eine kommerziell einsatzfähige Glühlampe bauen und vertreiben konnte, ohne diese Patente zu verletzen.

7 Goebel hatte zwei Patente im Zusammenhang mit der Glühlampenherstellung angemeldet, das Patent einer verbesserten Vakuumpumpe vom 5.1.1881 sowie das Verbesserungspatent über eine neue Verbindungsart von Kohlefäden mit Zuleitungsdrähten in der Glühlampe vom 23.1.1882. Nachdruck in Rohde (wie Anm. 2), S. 156-161.

8 Diese Meldung ist im Anhang von Rohdes Buch reproduziert, vgl. ebd., S. 238

9 Arthur Aaron Bright, The Electric-Lamp Industry. Technological Change and Economic Development from 1800 to 1947, New York 1949, S. 35-56; vgl. Rohde (wie Anm. 2), S. 23.

10 Thomas A. Edison, Electric lamp. US Patent 223.898, eingereicht am 4.11.1879, erteilt am 27.1.1880. 
Hatte sich Edison bis 1885 noch voll auf die technische Entwicklungsarbeit konzentriert, ging er nun gegen den unerlaubten Nachbau durch andere Firmen vor. Nach einem erfolgreichen Musterprozess gegen die United States Electric Lighting Company wurden einstweilige Verfügungen gegen andere unlizensierte Lampenhersteller beantragt, so auch gegen die Beacon Vacuum Pump \& Electrical Company in Boston. Diese erhob Einspruch und behauptete, Edisons Basispatent von 1880 sei ungültig, weil die Glühlampe zu diesem Zeitpunkt keine Neuheit mehr gewesen wäre. ${ }^{11}$ Bereits in vorangegangenen Prozessen hatte es Versuche gegeben, das grundlegende Patent anzufechten. Nun aber präsentierte das beklagte Unternehmen die Aussage einer Person, die bereits viele Jahre vor Edison eine Kohlefadenlampe hergestellt haben wollte: Henry Goebels Affidavit. ${ }^{12}$ Mehr noch, da die Original-Glühlampen oder zeitnahe Dokumente nicht mehr vorhanden waren, fertigte Goebel aus dem Gedächtnis Zeichnungen an, rekonstruierte Werkzeuge und stellte Lampen her.
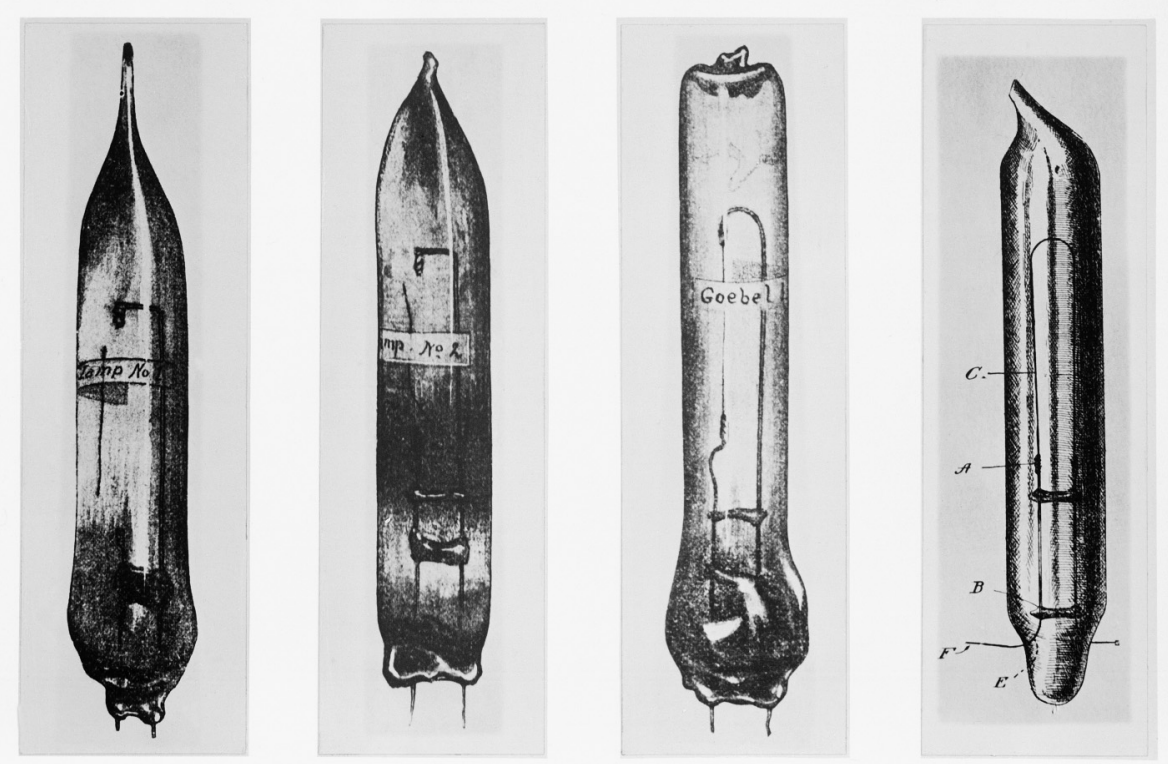

Abb. 2: Kohlefaden-Glühlampen von Henry Goebel, die bei den Verhandlungen als Beweismittel vorgelegt wurden. Quelle: Deutsches Museum, München.

In den Verhandlungen und Presseberichten fand diese Argumentation als „Goebel-Defence“ Eingang. Der geschilderten Verteidigungsstrategie der Beacon Vacuum Pump \& Electrical Company schlossen sich auch die Columbia Incandescent Lamp Company in St. Louis/Missouri und die Electric

11 Incandescent Lamp Litigation, in: Western Electrician 12, 1893, No. 5, S. 55.

12 Henry Goebel, in: The Electrical World 21, 1893, No. 18, S. 332. 
Manufacturing Company in Oconto/Wisconsin an. Die Anwälte dieser drei Unternehmen brachten die Goebel-Defence insgesamt in vier Prozesse ein. Teilweise nahmen diese Züge einer Gerichtsposse an. Die Parteien beschuldigten sich gegenseitig der Zeugenbestechung. So manche Aussage wurde später widerrufen. Angehörige der Goebel-Familie standen der Kläger-Seite zur Verfügung. So erklärte z.B. Henry Goebel jr., er und nicht sein Vater habe drei der als Beweis vorgelegten Lampen hergestellt - und zwar erst im September 1892. ${ }^{13}$ Detailliert geht Rohde in seiner Studie auf die jeweiligen Indizienketten der Anklage bzw. der Verteidigung ein und referiert die Entscheidungen der Richter sowie deren Urteilsbegründungen. Im Anhang sind sämtliche Affidavits aufgeführt - d.h. die schriftlichen Zeugenaussagen, anhand derer die Richter entschieden, können in deutscher Zusammenfassung nachgelesen werden.

Zum Verständnis der Ereignisse Anfang der 1890er Jahre sind noch folgende Zusammenhänge wichtig: Im April 1892 war aus dem Zusammenschluss der Edison Electric Light Company und der Thomson-Houston Electric Company die General Electric Company (GE) hervorgegangen. Die neue Gesellschaft entschied sich, hart gegen illegale Glühlampen-Produzenten vorzugehen. Insbesondere war man nicht bereit, diesen durch Lizenzgabe die Fortsetzung der Produktion zu erlauben. ${ }^{14}$ Zum einen stand GE unter Druck seitens der Lizenznehmer, das vertraglich zugesicherte Vertriebsmonopol auch durchzusetzen. Zum anderen hatte man die illegalen Produzenten zwölf Jahre lang unbehelligt gewähren lassen und wollte nun noch in den zwei Jahren bis zum Auslaufen des Patentschutzes versuchen, die enormen Entwicklungskosten über Gewinne zu realisieren. Eigentlich hatte ein USPatent eine Schutzfrist von 17 Jahren. Wurde aber die Erfindung in einem anderen Staat mit einer kürzeren Frist angemeldet, galt diese. Nun war die im grundlegenden US-Patent 223.898 von 1880 anerkannte Erfindung auch in Kanada angemeldet worden und dort lief der Schutz am 17. November 1894 aus. Man kann also - so Rohde - mit einigem Recht vermuten, clevere Anwälte hätten sich die Strategie ausgedacht, durch allerlei Winkelzüge den Prozess so lange zu verschleppen, bis der Patentschutz ausgelaufen sei. Für die verklagten Unternehmen war diese Strategie ohne Alternative, denn eine nachträgliche Lizenzvergabe hatte GE ausgeschlossen.

Unter diesen Umständen avancierte der inzwischen über 70-jährige Henry Goebel zum Hauptzeugen der Verteidigung. Er behauptete in einem Affidavit, ${ }^{15}$ bereits vor 1873 eine elektrische Glühlampe hergestellt zu haben. Vorarbeiten habe er in Springe unter Anleitung von Professor Münchhausen

13 The Oconto Incandescent Lamp Case, in: The Electrical World 22, 1893, No. 3, S. 45-50 u. No. 4, S. 68-72, besonders S. 46.

14 Rohde (wie Anm. 2), S. 33.

15 Das grundlegende Goebel-Affidavit vom 21.1.1893 ist im Anhang abgedruckt, vgl. ebd., S. 163-171. 
durchgeführt. Weiterhin sagte Goebel aus, er habe in New York zunächst mit Bogenlicht experimentiert und sogar eine Lampe auf das Dach seines Hauses montiert, was wiederum eines Nachts die Feuerwehr auf den Plan gerufen habe. Seine erste Glühlampe habe er aus einer Kölnischwasser-Flasche hergestellt, in die Platindrähte eingeschmolzen waren. Den Kohlefaden habe er aus verschiedenen Pflanzenfasern gewonnen und sei dabei auf Bambusfasern gestoßen. Das nötige Vakuum habe er nach dem Torricelli-Verfahren gewonnen; bei diesem Verfahren wurde der Lampenkolben mit Quecksilber gefüllt und dann gewendet, das herauslaufende Schwermetall hinterließ einen luftleeren Raum. An Goebels Aussage fällt auf, dass er auf alle drei Ansprüche in Edisons Basispatent einging. Weiterhin beschrieb er, dass er die Lampen zur Beleuchtung einer Uhr im Schlafzimmer, zur Illumination eines Schaufensters sowie seines Fernrohrs benutzt habe.

Die vier Prozesse wirbelten beträchtlichen Staub auf und in der amerikanischen Fachpresse erschienen ausführliche Berichte. ${ }^{16}$ Im ersten Verfahren entschied Richter Colt in Boston im Februar 1893, ,dass es extrem unwahrscheinlich sei, dass Henry Goebel 1854 eine praktisch einsatzfähige Glühlampe konstruiert habe“". ${ }^{17}$ Damit hatte sich Edison durchgesetzt und dem beklagten Unternehmen wurde die Produktion unlizensierter Lampen untersagt. Die Beacon Vacuum Pump \& Electrical Company gab aber nicht auf, sondern brachte eine Lampe auf den Markt, bei der die Zuleitungen nicht in den Glaskörper eingeschmolzen waren, sondern durch einen Stöpsel geleitet wurden. Damit hatte man zwar eine Bestimmung des Basispatents umgangen, weil aber das Schutzrecht für den Kohlefaden im Vakuum weiterhin verletzt war, wurde ein neues Verfahren angestrengt.

Im April 1893 wurde in St. Louis in der Sache Edison Electric Light Company/GE gegen die Columbia Incandescent Lamp Company verhandelt. ${ }^{18}$ Dabei hatte die Verteidigung auch die Erklärungen und Beweismittel aus dem Bostoner Verfahren eingebracht. Am 21. April 1893 verkündete Richter Hallett seine Entscheidung: Er wies den Antrag auf einstweilige Verfügung gegen die Columbia Incandescent Lamp Company ab, erließ jedoch die Auflage, dass das beklagte Unternehmen eine Kaution von 20.000 \$ für den Fall zu hinterlegen hätte, dass in einem späteren Verfahren und nach dem Kreuzverhör der Zeugen zugunsten der Klägerin entschieden werde. Dieser Spruch war eine herbe Niederlage für die Inhaberin der Edison-Patente, deren Monopol nun, 17 Monate vor dem Auslaufen des Patentschutzes im November 1894, rechtskräftig durchbrochen wurde. Da eine Revision nicht möglich war, gab es für GE nur zwei Möglichkeiten: Entweder man

16 So ist der Prozessverlauf in den amerikanischen Fachzeitschriften wie Electrical World oder Western Electrician weitgehend dokumentiert.

17 Rohde (wie Anm. 2), S. 38.

18 Incandescent Lamp Litigation, in: Western Electrician 12, 1893, No. 16, S. 207 u. No. 17, S. $220 \mathrm{f}$. 
ließ die Klage fallen, was der Unternehmensstrategie widersprochen hätte, oder man versuchte, die Angelegenheit in einem weiteren Verfahren, bei dem die Zeugen persönlich geladen und ins Kreuzverhör genommen werden konnten, endgültig zu klären. Letztlich fand eine solche Hauptverhandlung aber nicht mehr statt, da der Patentschutz im November 1894 ausgelaufen und damit einer Klage der Gegenstand abhanden gekommen war. Folgt man der Annahme, dass Goebel von den Anwälten der beklagten Seite lediglich benutzt worden war, um die Prozesse möglichst lange zu verschleppen, dann hat die Strategie zumindest in diesem einen Fall Erfolg gezeitigt.

Anfang Juli 1893 kam es in Milwaukee zu einer Anhörung in der Sache Edison gegen die Electric Manufacturing Company, Oconto. Der zuständige Richter entschied hier - wie zuvor Richter Colt in Boston - für die einstweilige Verfügung. Die beklagte Gesellschaft strengte eine Revisionsverhandlung an, die sie aber im Mai 1894 vor dem Appellationsgericht in Chicago verlor.

Rohde kommt nach Prüfung der Goebel-Defence zu dem Schluss: Keiner der Richter hatte in den vier Entscheidungen Goebel als Erfinder der elektrischen Kohlefadenlampe anerkannt. Nur die Columbia Incandescent Lamp Company hatte sich in St. Louis mit Hilfe der Goebel-Defence durchsetzen können. Aber auch der zuständige Richter Hallett hatte erhebliche Zweifel und machte deshalb der beklagten Company zur Auflage, 20.000 \$ zu hinterlegen. Rohde argumentiert, Richter Hallett wäre aufgrund einer etwas anderen Rechtsauffassung zu seinem abweichenden Urteil gekommen. „Während die anderen Richter klar fundierte Beweise forderten, um zur Durchsetzung der Rechtsansprüche eines Patents eine einstweilige Verfügung zu verweigern, genügten Hallett bereits Zweifel an der Berechtigung des Patents. “19 Das bedeutete aber keineswegs die Anerkennung von Goebels Priorität als Erfinder der Glühlampe und schon gar nicht die gerichtliche Feststellung eines Erfindungsdatums, ging es doch um finanzielle Interessen von Unternehmen und nicht um die Bestätigung einer Erfinderehre.

\section{Eine Legende entsteht: Ein Deutscher erfand die Glühlampe}

Für den Historiker ist nun interessant, wie aus der geschilderten Sachlage eine Legende werden konnte. Dies gilt umso mehr, weil das gesamte Verfahren in der amerikanischen Fachpresse ausführlich dokumentiert und die einschlägigen Zeitschriften auch in Deutschland gelesen wurden. Rohde geht davon aus, dass die Legende maßgeblich von Franklin L. Pope lanciert wurde. Der Patentanwalt und Fachjournalist für Elektrotechnik war eine Hauptstütze der Verteidigung. Außerdem hatten Pope und Edison in den Jahren 1869/70 sehr eng zusammengearbeitet, sich dann aber zerstritten. Popes Abneigung gegen Edison scheint als Motiv plausibel. Weiterhin kann vermutet werden, dass er sich seinen technischen Sachverstand von den beklag-

19 Rohde (wie Anm. 2), S. 47. 
ten Unternehmen gut bezahlen ließ. Dies ist im Detail nicht zu beweisen, aber einige Fakten sprechen für diese These: Bereits vier Tage nach Goebels schriftlicher Aussage für das Gericht publizierte Pope dessen Geschichte. ${ }^{20}$ Interessant sind dabei einige Änderungen zu Goebels Erklärung, anhand derer sich der Quellenbezug späterer Veröffentlichungen leicht feststellen lässt. So änderte Pope den Namen von Goebels wissenschaftlichem Mentor Münchhausen in Mönighausen, der Vater wurde aufgewertet, und zwar zum Konsul der Niederlande in New York in den Jahren 1819 bis 1820 und mit der Formulierung ,in picturesque English with a strong Teutonic flavor" unterstellte er dem Einwanderer schlechte englische Sprachkenntnisse. ${ }^{21}$ Letzteres sollte erklären, warum dieser sich die Erfindung der Glühlampe nicht sofort hatte patentieren lassen. Im Februar 1893 wurde die GoebelGeschichte in Deutschland von der Elektrotechnischen Zeitschrift (ETZ) bekannt gemacht - und zwar als zusammenfassende Übersetzung von Popes Artikel. ${ }^{22}$ Einige Wochen nach der Zurückweisung der Goebel-Defence durch Richter Colt in Boston berichtet die ETZ erneut, diesmal skeptischer. Die Meldung schließt mit den Worten: „Das Ganze erinnert an die ähnlichen Versuche, das Bell-Telephonpatent anzugreifen, und die romantische Geschichte des armen Erfinders Goebel wird bald der Vergessenheit anheimfallen." ${ }^{23}$

1911 ging der Ingenieur Lothar Arends aus Berlin auf die Suche nach Details und publizierte diese in einer von Franz Maria Feldhaus herausgegebenen Zeitschrift. ${ }^{24}$ Allein, den Stein ins Rollen brachte ein 1923 von Hermann Beckmann, Abteilungsvorstand der Accumulatoren-Fabrik AG in Berlin, veröffentlichter Artikel in der ETZ. ${ }^{25}$ Dieser beruhte auf dem Artikel von Pope sowie auf den Beiträgen in der ETZ von 1893. Beckmann nahm auch Bezug auf den kritischen Beitrag von Tanner ${ }^{26}$ dessen Zweifel fanden

20 Franklin Leonard Pope, The Carbon Filament Lamp of 1859- The Story of an Overlooked Invention, in: Electrical Engineer 15, 1893, No. 247, S. 77-80; abgedruckt im Anhang von Rohde (wie Anm. 2), S. 240-243. A.M. Tanner stieß 1894 bei seiner Recherche vor Ort auf die Aussagen des langjährigen Professors an der TH Hannover Christian Moritz Rühlmann: ,that no person by the name of Moenighausen, or Munchhausen, was ever known as a ,professor" in the kingdom of Hanover", s. A.M. Tanner, The GoebelMunchhausen [sic] Lamp Story, in: The Electrical Review 34, 1894, No. 845, S. 113f., hier S. 113.

21 Rohde (wie Anm. 2), S. 83.

22 [K.W.] Der Erfinder der Glühlampe, in: ETZ 14, 1893, H. 7, S. 89f.; auch [W.] Heinrich Goebel, der Erfinder der Glühlichtlampe, in: Elektrotechnische Rundschau 19, 1892/93, H. 12, S. 101. Hier heißt es unmissverständlich: „Es ist festgestellt, daß ein Deutscher, Namens Heinrich Goebel, [...] der Erfinder der Glühlampe ist.“

23 [P.T.] Der Glühlampenpatentstreit in Amerika, in: ETZ 14, 1893, H. 14, S. 206.

24 Lothar Arends, Heinrich Goebel, der Vater der elektrischen Lichtreklame, in: Geschichtsblätter für Technik, Industrie und Gewerbe 2, 1915, Nr. 3/4, S. 114f.

25 H. Beckmann, Die erste Glühlampe, in: ETZ 44, 1923, H. 47/48, S. 1031-1034.

26 Tanner (wie Anm. 20). Dieser Beitrag wurde referiert: English View of the Incandescent Lamp Situation, in: Western Electrician 12, 1893, No. 23, S. 306. 
aber keinen Eingang in den ETZ-Aufsatz. Rohde erklärt dies mit dem Selbstverständnis der Ingenieure: „Als Techniker vertraute er [Beckmann - FD] offenbar wie Arends auf die Richtigkeit der Darstellung seines amerikanischen Techniker-Kollegen Franklin L. Pope [...] Widersprüche wurden nicht kritisch problematisiert, sondern spekulativ harmonisiert ". ${ }^{27}$ Beckmann arbeitete auch nachlässig. So übernahm er nicht das korrekte Todesdatum, den 4. Dezember, sondern gab stattdessen den Erscheinungstag des Nachrufs in der Electrical World an. ${ }^{28}$ Auf diese Weise kam der 16. Dezember 1893 als Todestag in fast alle deutschen Publikationen über Goebel. Fast möchte man Beckmann dafür danken, da auf diese Weise die Herkunft späterer Angaben leicht nachvollziehbar wird.

Rohde erklärt Beckmanns unkritische Arbeitsweise auf zwei Ebenen. ${ }^{29}$ Beckmann hatte Verwandte in Springe und deshalb auch persönliches Interesse an der Geschichte. Als zweiten Grund führt Rohde die patriotische Grundstimmung in Deutschland nach dem Versailler Vertrag an, der von weiten Kreisen als demütigend empfunden wurde. Nun war nationales deutsches Heldentum gefragt. Der Autor stützt seine These mit dem Hinweis darauf, dass Beckmann auch einen anderen Deutschen aus der Versenkung gehoben hatte, nämlich Johann Wilhelm Ritter als Entdecker des Grundprinzips des Akkumulators.

Über Beckmanns Beitrag fand Goebel Eingang sowohl in Nachschlagewerke, so u.a. in Conrad Matschoss' Männer der Technik sowie in Meyers Lexikon von 1926 und in den Brockhaus von 1930, als auch in Fachartikel. Einen ersten Höhepunkt hatte die Goebel-Glorifizierung 1929 im Zusammenhang mit den Feierlichkeiten zum 50-jährigen Jubiläum der Erfindung der Glühlampe durch Edison in den USA. Im Sinne einer nationalen Korrektur wurde massiv auf den deutschen Erfinder hingewiesen und auf Initiative der Elektrotechnischen Gesellschaft Hannover am Geburtshaus in Springe eine Gedenktafel enthüllt. Der Festredner war Hermann Beckmann, „dem das Verdienst zukommt, den Namen Heinrich Goebels der Vergessenheit entrissen zu haben" ${ }^{30}$ der in seiner Ansprache aber auch die Verdienste anderer Erfinder anerkannte. ${ }^{31}$

Die Legende eines deutschen Erfinders der Glühlampe passte gut in die nationalsozialistische Zeit. ${ }^{32}$ Besondere Aktivitäten konnte Rohde aber erst

27 Rohde (wie Anm. 2), S. 90.

28 Death of Henry Goebel, in: The Electrical World 22, 1893, No. 25, S. 456; auch Henry Goebel, in: Western Electrician 13, 1893, No. 25, S. 307f. (beide vom 16.12.1893).

29 Rohde (wie Anm. 2), S. 91

30 Heinrich-Goebel-Feier in Hannover, in: ETZ 50, 1929, H. 37, S. 1349f. Zitat aus [Schp.] Heinrich-Goebel-Feier in Springe am Deister, in: ebd., H. 41, S. 1492.

31 „Dr. Beckmann betonte, daß die nachträgliche Anerkennung und Ehrung Heinrich Goebels in keiner Weise die durch Swan und Edison erworbenen Verdienste um die Einführung der von ihnen gebauten Glühlampen als Lichtquelle schmälern könne.“ (s. ebd.).

32 Möglicherweise spielte hier auch Goebels gemeinsamer Geburtstag mit Hitler am 20. April eine Rolle. 
1938 im Vorfeld von Goebels 120. Geburtstag nachweisen. Nachdem anlässlich des 90. Geburtstags von Edison 1937 in Menlo Park ein großes Glühlampensymbol errichtet worden war, sollte nun auch Goebel in Springe gebührend geehrt werden. In Presseberichten dieser Zeit wird die Legende ausgeschmückt. So wuchs z.B. die Brenndauer der ersten Lampe gegenüber Beckmanns Angaben auf das Zehnfache - 4000 Stunden. Immer mehr erscheint Goebel als „das typische Beispiel eines deutschen Erfinders“, der sich ,eine Idee in den Kopf setzte, die der Zeit weit voraus eilte [...] und der nur auf sich selbst angewiesen an der Verwirklichung dieser Idee arbeitete“. Nach vollbrachter Tat „war er zufrieden und es kam ihm nicht in den Sinn, seine Erfindung mit großem Lärm anzupreisen und Kapital aus ihr zu schlagen, wie es sein großer Konkurrent tat" ${ }^{33}$ Osram ließ Goebels Glühlampen nachbilden. ${ }^{34}$ Auch in der Kriegszeit fand sich Gelegenheit zur Goebel-Ehrung, als sich 1943 der Geburtstag zum 125. Male und der Todestag zum 50. Mal jährte. Nach der Kriegserklärung an die USA wurde der Antiamerikanismus stärker. Goebel wurde zum edlen Erfinder und Idealisten, der sich vom typisch amerikanischen Materialismus abhob und obendrein noch ,wertvolles deutsches Blut" als „Kulturdünger“" in die USA gebracht habe. ${ }^{35}$

Nach Ende des Zweiten Weltkriegs erlebte die Goebel-Legende zunächst eine Wiederholung in Variationen. Der damalige Leiter der Stadtverwaltung Springe Gustav Degenhardt engagierte sich hier besonders. Immer wenn in der Presse von Edisons Leistungen die Rede war, meldete er in Form von Leserbriefen Korrekturen an. Neben Patriotismus war wohl auch ein gezieltes Stadtmarketing das Motiv. Nach Degenhardts Pensionierung flaute in den 1970er Jahren diese „Erinnerungsarbeit“ ab. 1993 bot sich mit dem 175. Geburtstag und dem 100. Todestag erneut Gelegenheit, auf den deutschen Erfinder der Glühlampe zu verweisen. 2004 folgte eine wiederum kritiklose Würdigung anlässlich der Jubiläumsfeier „150 Jahre Glühlampe“ in Springe, begleitet von einer publikumswirksamen Berichterstattung im Fernsehen sowie einer Sonderbriefmarke.

\section{Fazit}

Rohde legt mit seinem Buch einen spannenden Gerichtsreport vor, in dem er schlüssig argumentiert. Mit Akribie geht er jenen Spuren nach, die heute in den Archiven noch auffindbar sind. Detailliert schildert er die verschiedenen Verhandlungen, die von der Anklage bzw. Verteidigung eingebrachten Be-

33 Alle Zitate [Hg.] Heinrich Goebel, in: Funk 1938, H. 4, S. 103f., hier S. 104.

34 Das Deutsche Museum hatte bereits im Dezember 1926 bei dem thüringischen Glasbläser Emil Gundelach „elektrische Glühlampen der Vor-Edison'schen Zeit“ in Auftrag gegeben. Gundelach fertigte im Januar 1927 nach Zeichnungen und Fotos des Deutschen Museums neben anderen auch drei „Glühlampen nach Göbel (1854)“ an (Inventar-Nr. 59292).

35 Rohde (wie Anm. 2), S. 103. 
weismitteln sowie die Entscheidungen und Urteilsbegründungen der Richter. Mit gleicher Akribie beschreibt er die Aktivitäten rund um die GoebelWürdigung in der Stadt Springe bis in die Gegenwart. Im 100-seitigen Anhang sind Patentschriften, zentrale Zeitschriftenartikel und Aussagen abgedruckt. Damit kann sich der Leser sein eigenes Urteil bilden. Und diese Form der Offenheit ist zweifellos zu begrüßen, denn die Emotionen kochen hoch in Springe. Selbst große deutsche Zeitungen haben dieses Thema aufgegriffen. ${ }^{36}$

Kurze Zeit keimte die Hoffnung auf, fern von aller Quellenarbeit und Textinterpretation den ultimativen Beweis pro oder contra die Goebel-Defence führen zu können. Im Frühjahr 2006 wurden nämlich im Henry-Ford-Museum in Dearborn jene Goebel-Lampen aufgefunden, die im Prozess als Beweismittel eingebracht worden waren. Aber auch das könnte sich als trügerische Hoffnung erweisen. Falls es nämlich die wirklichen „Goebel-Lamps“ wären, der heutige Eigentümer einer Untersuchung zustimmte und das Geld für eine Analyse aufgebracht würde, ist doch unklar, ob eine Differenz von etwa 25 Jahren an den verkohlten Bambusfäden nachweisbar wäre. Eigentlich kann man sich nur ein eindeutiges Ergebnis vorstellen, nämlich dass der Bambus nach 1880 geschlagen wurde. Dann hätte Edison sein Patent zu Recht. Ein Erntezeitraum vor 1880 dagegen wäre kein hinreichendes Argument für den nun gestürzten Erfinder, denn Goebel hatte nach eigenem Bekunden die Bambusfäden aus einem alten Bambusstock gewonnen. Prioritätsansprüche lassen sich also nicht allein mit naturwissenschaftlichen Methoden klären und Technikgeschichte nicht auf Erfindergeschichte reduzieren. Das dürfte die Technikhistorikerinnen und -historiker freuen, deren Arbeit auch weiterhin gebraucht wird.

Anschrift des Verfassers: Dr. Frank Dittmann, Deutsches Museum, Museumsinsel 1,80538 München, E-Mail: f.dittmann@deutsches-museum.de

36 Christian Gruber, Es werde Licht, in: Die Rheinpfalz am Sonntag vom 21.1.2007, S. 19; Michael Prellberg, Wem zuerst ein Licht aufging, in: Financial Times Deutschland vom 22.1.2007, S. 28; Heinrich Thies, Springe ohne leuchtendes Idol, in: Hannoversche Allgemeine Zeitung vom 22.1.2007, S. 3; ders., Heinrich Göbel. Der Mann, der die Glühbirne doch nicht erfand, in: Die Zeit vom 25.1.2007, S. 11; Willi Winkler, Meister Lampe, in: Süddeutsche Zeitung vom 22.2.2007, S. 9 (Panorama). 